\title{
DESIGN OF THE DATA TRANSMISSION BETWEEN THE EXERSICE SMART MINE WITH GROUND STATION USING LORA
}

\author{
Gesit Pratiknyo, M Sigit Purwanto, Erpan Sahiri, Muladiyono \\ Indonesia Naval Technology College, STTAL Surabaya Indonesia
}

\begin{abstract}
Wireless data communication system through communication radio is one of the important points of information delivery process in TNI AL, It relates to the process of delivering information on the field that is difficult to obtain communication network via internet. The use of portable and strong communications against jammed interference and other adjacent frequency interference becomes a fundamental necessity. The use of the UHF frequency communication system is one of the efficient communication, With the high frequency of positively resulting in the physical size of the antenna is increasingly smaller. The purpose of designing data communication system devices between exercise smart mine and the ground station using Lora. In planning this communication system uses the Lora SX1278 module as a data transmitter (Tx) and receiver data device (Rx). On the transmitter side (Tx) Lora uses the Arduino Mega 2560 microcontroller as the core or brain system. On the side of the receiver $(R x)$ Lora uses Arduino Uno R3 as the process data unit received by the Lora receiver. The main function of Arduino Uno as an intermediary to be able to transfer data to a PC or laptop device as a ground station monitoring through USB communication channels. From the design, testing, and discussion of research results during the preparation and manufacture of this final task obtained the results as expected. That the design of data communication systems using Lora can be obtained a maximum range of 200 meters with the condition of building obstructed. The ability of the Lora SX1278 module to receive data and transmit data from the GPS, pressure sensors and acoustic sensors is well received by the ground station, based on the data transmitted via the Lora SX1278 module transmitter.
\end{abstract}

Keywords: Lora SX1278, Arduino Mega 2560, Arduino Uno R3

\section{INTRODUCTION}

Indonesia is a country consisting of several islands where two thirds of its territory is the sea, Many marine flows of Indonesian archipelago that can be traversed by ships of other countries into Indonesian waters. To anticipate this in terms of State defense, need one of the tools that we need to prepare as a stronghold of the sea flows for the security of the country Indonesia.

In this case the $T N I A L$ is one element of the nation of Indonesia that has the task to defend the unity and unity of the Republic of Indonesia. In carrying out the task of sustaining nation sovereignty, TNI AL requires a lot of supporting devices, Supporting devices one of which is a supporting tool in carrying out security tasks. These supporting tools are modern and sophisticated supporting tools to overcome difficult problems. Supporting equipment may not be the main equipment in carrying out security tasks, But the supporting equipment provides significant usability in providing benefits.

Marine mines are one of the explosive devices placed in the waters to destroy enemy targets or targets, The water or submarine ship. The way the sea mines work will be active if the target enemy approaches the mines so it will trigger mines to explode. Marine mines can be run offensive by laying close to the target, or defensive by placing them at a safe location so that they are harmless to the personnel themselves or friends. To spread mines usually use warships (KRI) or $T N I A L$ personnel assigned specifically to this.

Wireless data communication system through communication radio is one of the important points of information delivery process in $T N I A L$, It relates to the process of delivering information on the field that is difficult to obtain communication network via internet. The use of portable and strong communications against jammed interference and 
other adjacent frequency interference becomes a fundamental necessity.

The use of the communication system using UHF frequency is one of the efficient communication, with the high frequency of positive resulting in the physical size of the antenna is increasingly smaller. The frequency of UHF 433 $\mathrm{MHz}$ is the frequency specified according to tropical Asia. While the frequency of $915 \mathrm{MHz}$ is a frequency destined for European and American regions.

In realizing the data communication system via radio communication is inexpensive and able to reach a distance far enough, The author tries to lift it into a research theme with the title of research Design of The Data Transmission Between The Exersice Smart Mine with Ground Station Using Lora.

.Based on background, Then the formulation of problems in this research is about how to design or design device data communication system between exercise smart mine with the ground station using Lora.

The purpose of this research is to design or design device data communication system between exercise smart mine and the ground station using Lora.

\section{RESEARCH METHODS}

\subsection{Design Research}

Design of The Data Transmission Between The Exersice Smart Mine with Ground Station Using Lora is a research that is expected to translate the analysis of a system into the communication system in the UHF network and make Communication system using Lora. In designing this author uses Lora at a frequency of $433 \mathrm{Mhz}$ referring to the use of a frequency of 433 Mhz corresponding to the Asian region.

To implement the research well, the preparation of steps to find the data source as well as conducting a research device Design of The Data Transmission Between The Exersice Smart Mine with Ground Station Using Lora which includes Research instruments, primary data, secondary data, and the validity and reliability testing of data.

\subsection{Time and place}

The research location is a place where the research will be conducted. The research conducted by the authors took the location at the campus STTAL Moro Krembangan Surabaya. The time used in this study began in July 2019 to the end of December 2019.

\subsection{Tools and Materials}

The equipment to be used in the research and design of this final task :
a. 2 unit Lora SX-1278
c. 1 unit Arduino Uno R3
e. Arduino IDE 1.8.0
f. 1 set toolset
g. 1 AVOmeter
h. 1 unit solder

b. 1 unit Arduino Mega 2560

d. Computer (PC) 32/64 bit architecture processor, 4 GB Random Access Memmory (RAM), Operation System windows 7

\subsubsection{Research Draft}

Designing will be easier in the realization of equipment with hardware block diagrams. In designing it is expected to clarify what systems and hardware devices used in this research. Following system block diagrams and flow charts on the design of this research:

a. System Block Diagram

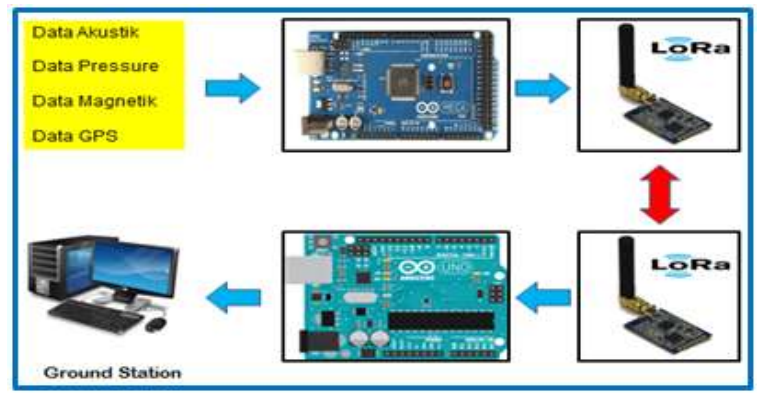

Fig.1 System Block Diagram 
The diagram block in Figure 1 above describes the design of the system as well as the data communication transmitted through the intermediary antenna $\mathrm{TX}$ or $\mathrm{RX}$ in Lora with a frequency of $433 \mathrm{MHz}$, which is used for data transmission from acoustic sensors, Pressure sensors, magnetic sensors and GPS between the Exercise Smart Mine mines located in the sea to ground station operators on the ground to be displayed on a computer (PC).

b. Research Stages Flowchart

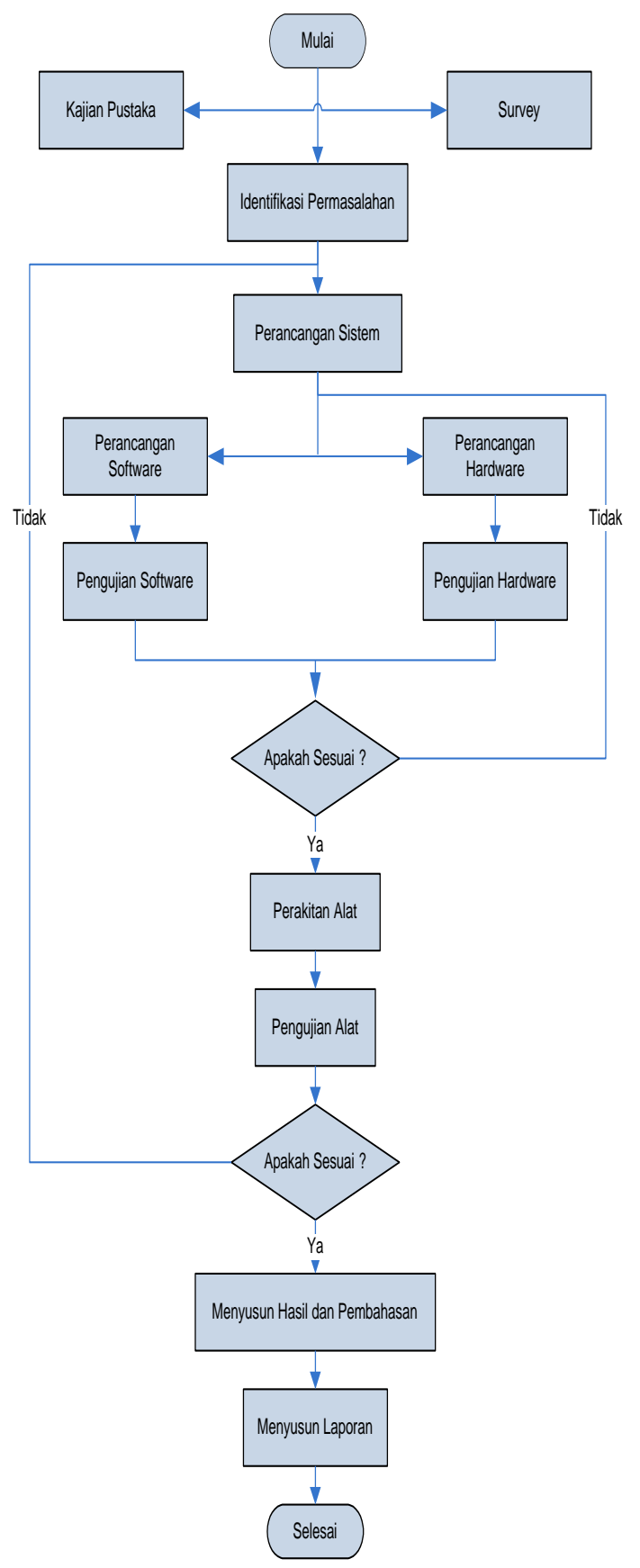

Fig.2 Research Stages Flowchart

\subsection{Data Collection}

On Design of The Data Transmission Between The Exersice Smart Mine with Ground Station Using Lora authors conducted a study by collecting Data. Research data collection Method design build Data delivery between Exercise Smart Mine with Ground Station using Lora will be done the author, is :

a. Interviews, this way by doing a question and answer to the competent party regarding the communication of data wirelessly.

b. Observation, this way the author is done by conducting research directly in the field.

c. Documentation, this way is done by collecting data from books, records, and research results in related agencies on data communications wirelessly.

\subsection{Data processing}

Data processing is a process by which authors post some information collected on the data collection process. The Data obtained will be conducted and become reference to the design and system design analysis process.

The operational definition of this design plan explains and defines the variables used in this design plan. The following operational definitions in the design of the, is :

a. Frequency : A unit of measurement of the number of carrier frequencies in one second with $(\mathrm{Hz})$.

b. Decibel : A unit of carrier signal strength on the data communication system via radio frequency $(\mathrm{dB})$.

c. BaudRate : Data delivery format in bits per second (bps).

\section{DESIGNING, IMPLEMENTING AND TESTING THE SYSTEM}

\subsection{System Design}

In the design analysis of data communication system using Lora that will be created authors will describe the design of the system created, the data 
transfer format and the way to process the data that has been received on the receiver side. The data Format used in the delivery process is compiled according to the data requirements on the receiver side.

\subsubsection{Transmitter Lora System Design}

In Figure 3, the following diagram of the Lora transmitter system blocks, in charge of collecting data from GPS, pressure sensors and acoustic sensors in the marine Exercise Smart Mine.

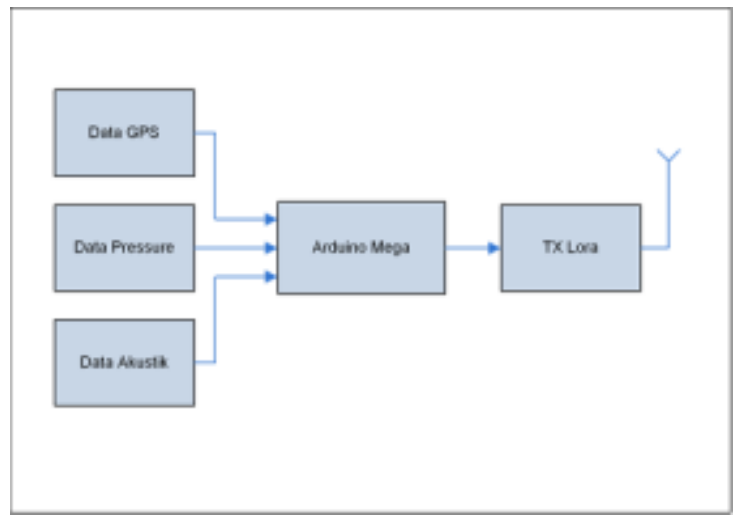

Fig.3 Transmitter Block System Lora

In designing the sender data format is compiled as information based on data from GPS, pressure sensors and acoustic sensors. Each data will be sent to Lora receivers alternately or is a queue system. Data from sensor devices received by Lora's transmitter system will be sent the first time so next to another sensor.

The preparation of the data to be sent by the transmitter :

Data = latitude + ', + longitude + ', + satelite + ', + hdop + ', + altitude + ', + speed + ', + date + ', + time + ', + pressure + ', + acoustic.

The Data is compiled with a delimiter character (,) comma as an identifier when it poses parsing on the receiver.

On-side transmitter (Tx) Lora authors use Arduino Mega microcontroller 2560 as the core or brain system. The authors use this microcontroller because of the need for a serial communication of 4 (four) series. Each of these series is needed to communicate with GPS, acoustic, pressure and serial devices to communicate with Lora devices.

To draw an integrated Lora transmitter system with other sensor devices can be viewed in the following Figure 4 transmitter device integration

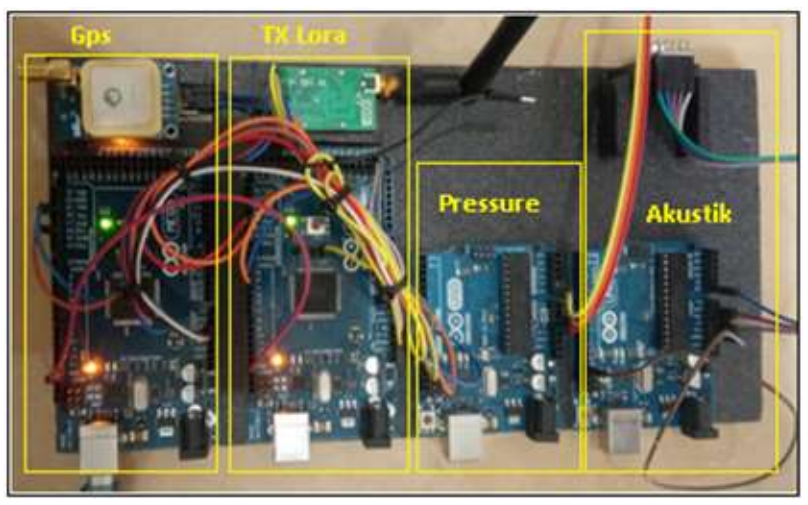

Fig.4 Transmitter System Integration

\subsubsection{Designing the Receiver Lora System}

On the receiver side, authors use Arduino Uno as a data processing unit received by Lora receivers, the main function of Arduino Uno as an intermediary to be able to transfer data to a PC or laptop device as a Ground Station Monitoring Through a USB communication channel. To illustrate the receiver system can be seen on the diagram, the following receiver block :

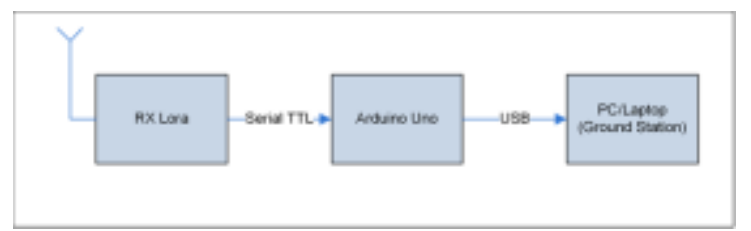

Fig.5 Receiver System Block Diagram

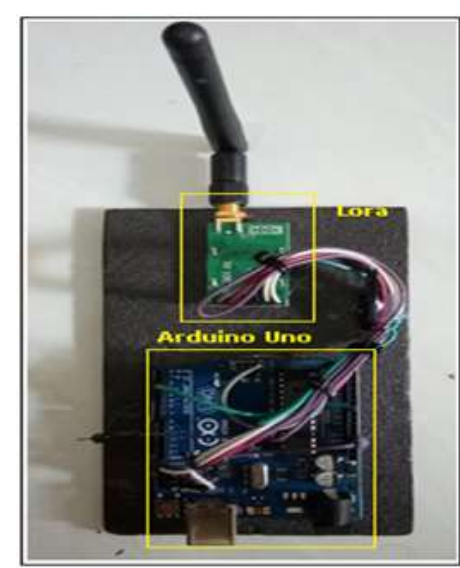

Fig.6 Receiver Network Physical Form 


\subsection{Implementation of Research}

Implementation of this research is the implementation of the planning and designing that has been arranged in detail, is expected to be implemented maturely and according to the results of the expected research. Phase implementation of data communication system using Lora on the draft that has been made are as follows.

\subsubsection{Transmitter Lora Implementation}

The transmitter is used to transmit the data of all integrated sensors in the mine system, the implementation of sensor data must be arranged to allow the recipient to know the data received in accordance with the intent and purpose of the data. GPS sensor data begins with the GPS sentence:, Pressure sensor data begins with PRESS character: and acoustic data begins with ACC: character.

\subsubsection{Receiver Lora Implementation}

In its implementation on the ground station side can do the separation of data received by Lora receivers. The separation process is called also by another term which is parsing. Process of separating data based on the initial character that has been established on the transmitter side. Detection process by detecting the initial GPS:, PRESS: and ACC: Characters.

The next data that follows the original character will be split to get the information contained, the split process is done according to the data separator character (' , '). The process of splitting the data on ground station systems can be viewed in the following C\# program code :

if (data.Contains("GPS:"))\{

String D_GPS = data.Replace("GPS:", "');

String [] parse = D_GPS.Split(',');

\}else if (data.Contains("PRESS:"))\{

String D_PRESS = data.Replace("PRESS:",

"');

String [] parse $=$ D_PRESS.Split(',');

\}else if (data.Contains("ACC:"))\{
String D_ACC = data.Replace("ACC:", "');

String [] parse = D_ACC.Split(',');

\subsection{System Testing}

Testing the research was conducted to obtain the expected data. Some of them are testing data acceptance. Testing the acceptance of data from GPS emitted via the Lora transmitter is then accepted by the Lora receiver displayed via the PC monitor screen.

\subsubsection{Testing of Data Delivery and Acceptance}

To be able to test data transmission then the transmitter device and need to be connected as in Figure 7 below :

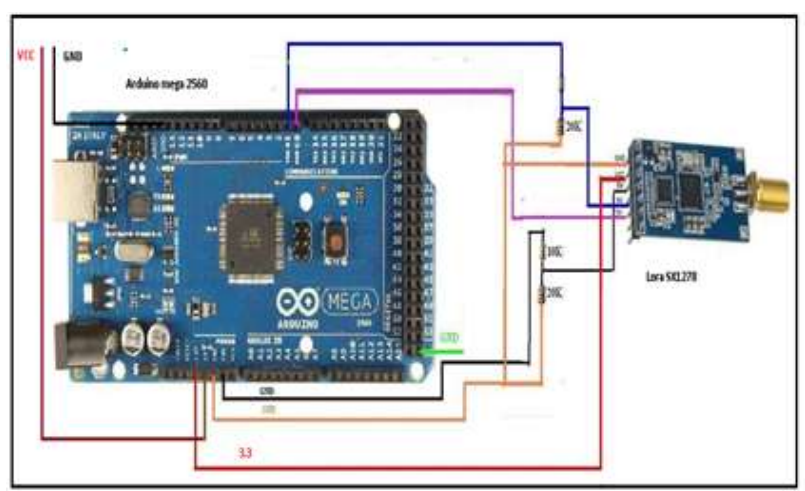

Fig.7 Wiring Diagram Device Transmitter

The next step is to upload a program that has been created to Arduino Mega 2560 device. This code is a code or program listing for a transmitter device.

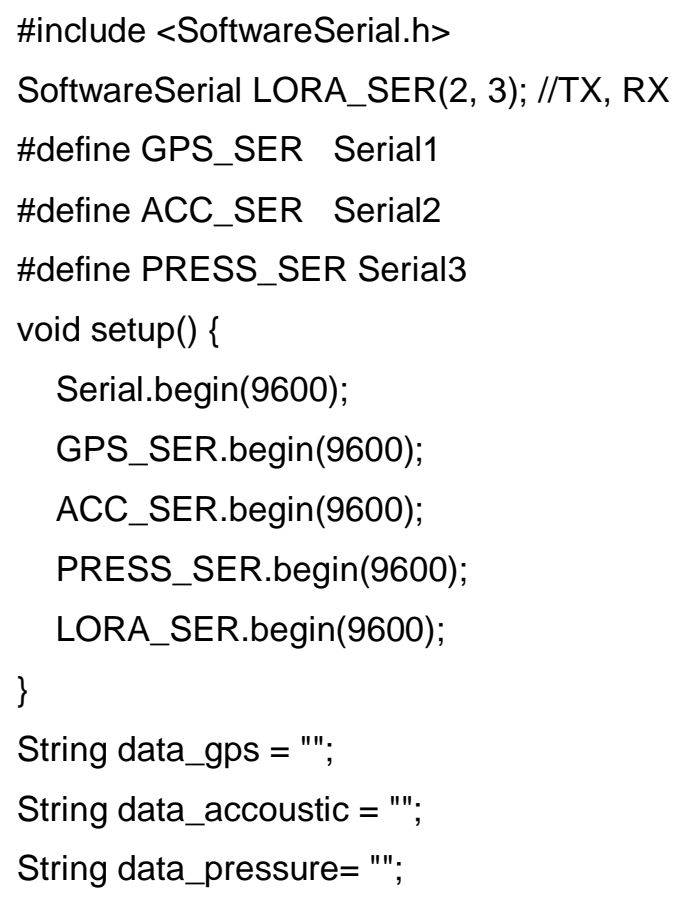


void loop() \{

$$
\begin{aligned}
& \text { if(GPS_SER.available }()>0)\{ \\
& \text { data_gps }=
\end{aligned}
$$

GPS_SER.readStringUntil('In');

data_gps.trim();

Serial.print("GPS:");

Serial.println(data_gps);

LORA_SER.print("GPS:");

LORA_SER.println(data_gps);

\}

if(ACC_SER.available ()$>0)\{$

data_accoustic $=$

ACC_SER.readStringUntil('In');

data_accoustic.trim();

Serial.print("ACC:");

Serial.println(data_accoustic);

LORA_SER.print("ACC:");

LORA_SER.printIn(data_accoustic);

\}

if(PRESS_SER.available ()$>0)\{$

data_pressure =

PRESS_SER.readStringUntil('In');

data_pressure.trim();

Serial.print("PRESS:");

Serial.println(data_pressure);

LORA_SER.print("PRESS:");

LORA_SER.printIn(data_pressure);

\}

\}

To be able to test data acceptance then the receiving device and need to be connected as in Figure 8 below :

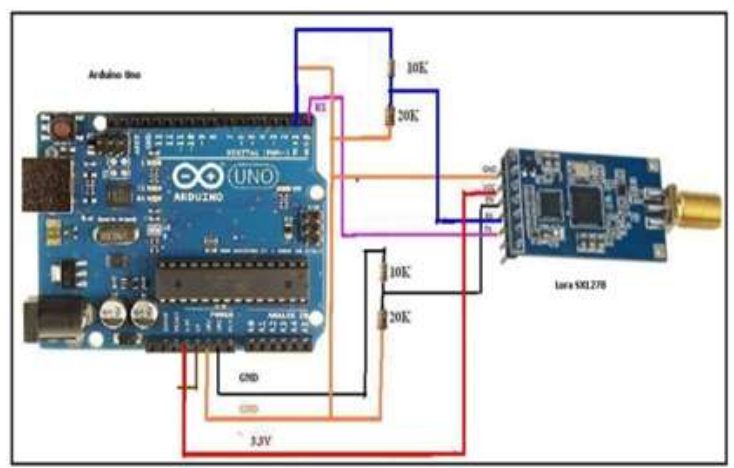

Fig.8 Wiring Diagram Device Receiver
The next step is to upload a program that has been created to the Arduino Uno device. This code is a code or program listing for the receiver.

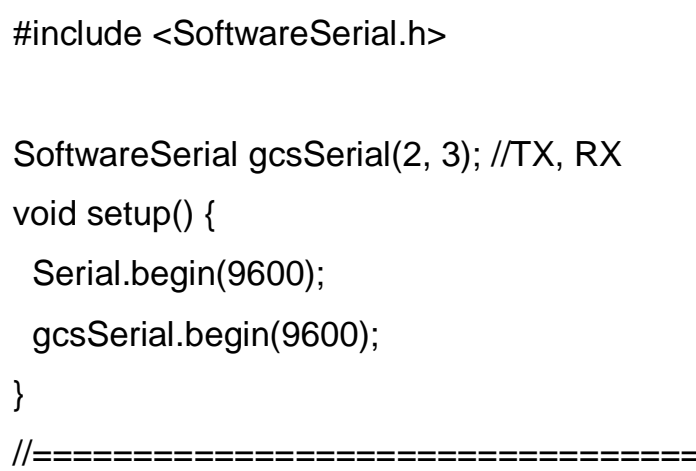

unsigned long timer;

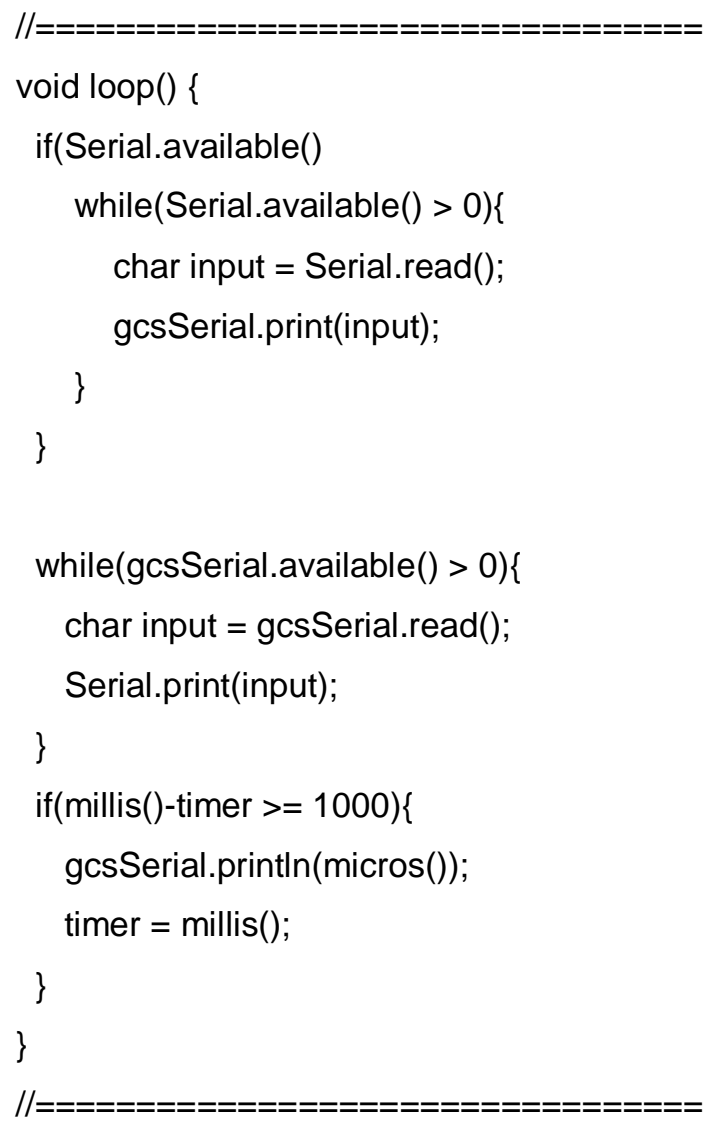

\subsubsection{Testing Program Display on Ground Station}

After all the tests have been uploaded to each microcontroller then the next stage is connecting the GPS device, acoustic sensor and the sensor device to a pressure to Arduino Mega. Arduino Uno device connected with Lora receiver connected with PC or laptop using USB cable and running Ground Station program. 


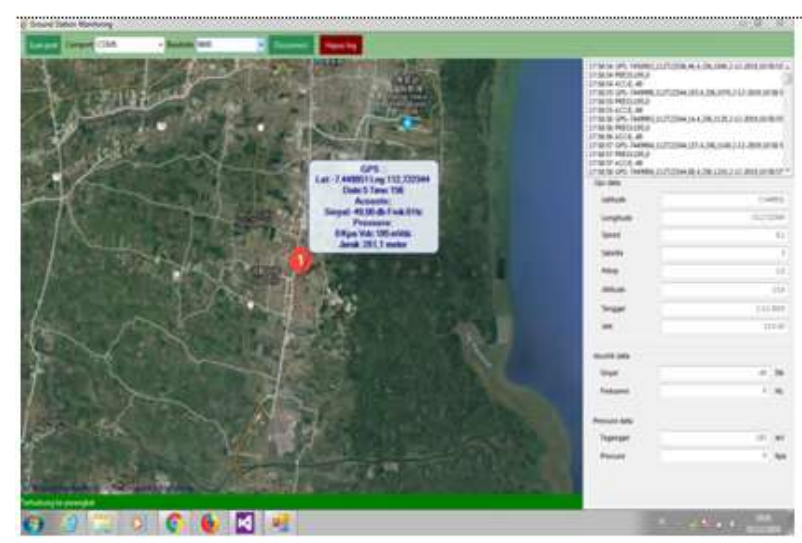

Fig.9 Ground Station Program View

In Figure 9 above is a view of the Ground Station program created by the authors using the C\# programming language. The Ground Station Program can display location data in the form of map visualizations and data from received sensor devices.

From the data received will be done the process of splitting the data according to the implementation phase of data separation, data separation results will be displayed in the program interface Ground Station. Program code to perform splitting of data or parsing data received by the recipient can be seen the following program code :

if (data.Contains("GPS:")) \{

String D_GPS = data.Replace("GPS:", "');

String [] parse = D_GPS.Split(',');

if $($ parse.Length $==8)\{$

double latitude $=$

Convert.ToDouble(parse[0]) / 1000000;

double longitude $=$

Convert.ToDouble(parse[1]) / 1000000;

txtLat. Text $=$

String.Format("\{0:0.\#\#\#\#\#\}",Convert.ToDouble(par se[0]) / 1000000);

$$
\text { txtLng. Text }=
$$

String.Format("\{0:0.\#\#\#\#\#\}",

Convert.ToDouble(parse[1]) / 1000000);

txtSpeed.Text = String.Format("\{0:0.\#\}",

Convert.ToDouble(parse[2]) / 100);

txtDate. Text $=$ parse[6];
txtTime. Text $=$ parse[7];

txtSat.Text $=$ parse[3];

txtHdop.Text = String.Format("\{0:0.\#\}",

Convert.ToDouble(parse[4]) / 100);

txtAltitude.Text $=$

String.Format("\{0:0.\#\}", Convert.ToDouble(parse[5]) / 100);

Once the program is connected to the receiver device through a serial communication, the ground station interface will display the data received based on the data the transmitter sent. Here is the data acceptance interface view :

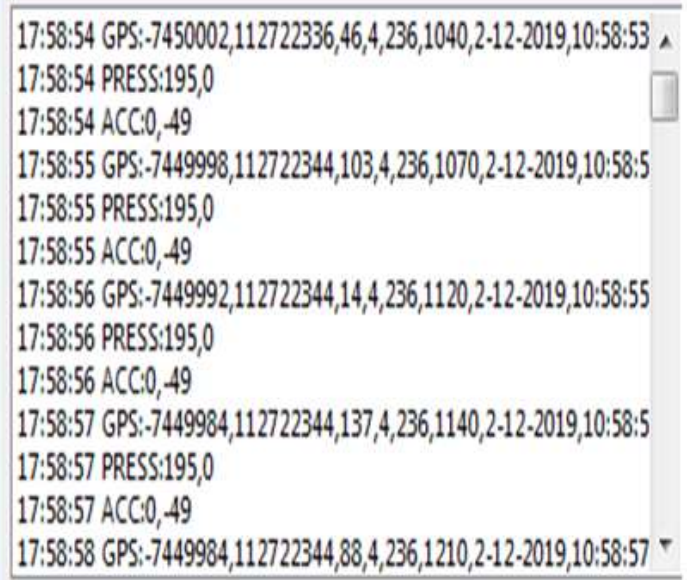

Fig.10 Data Acceptance View

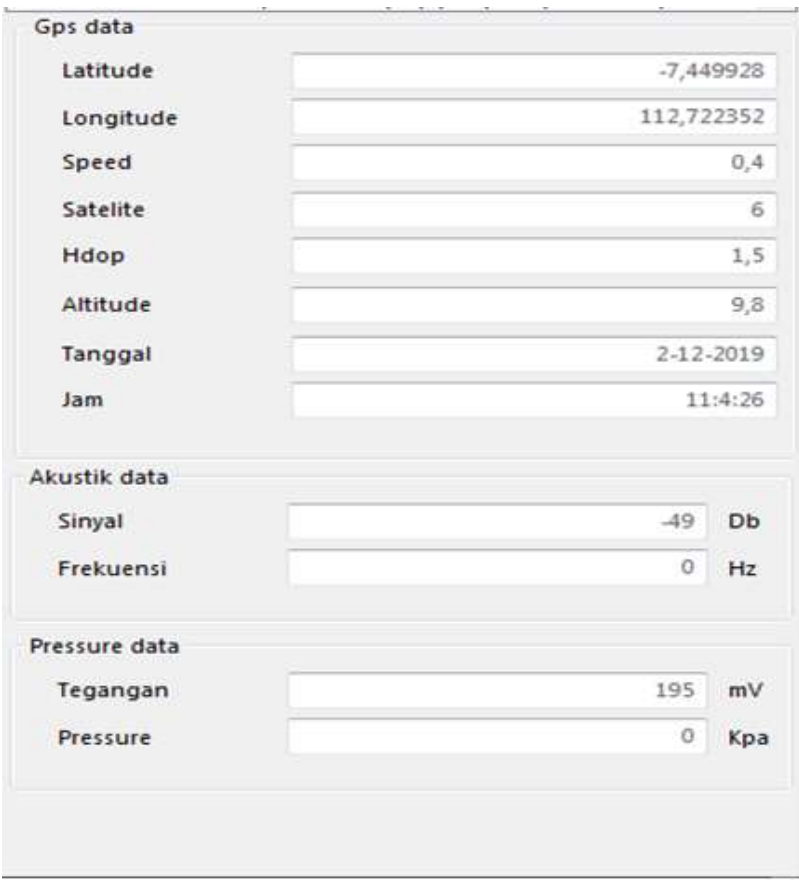

Fig.11 GPS Data Display, Acoustic Sensors and Pressure Sensors 
To display GPS data on an electronic map can be seen in the following program code :

gMapControl1.Overlays.Remove(ranjauOverl ay);

if(ranjauOverlay.Markers.Count() $>0)$ ranjauOverlay. Clear();

gMapControl1.Overlays.Add(ranjauOverlay);

GMarkerGoogle markerNode $=$ new

GMarkerGoogle(new PointLatLng(latitude,
longitude), bitmap);

GMap.NET.PointLatLng point $=$ new PointLatLng(latitude, longitude);

markerNode.Position = point;

markerNode.ToolTipText = "GPS :: InLat:" + String.Format("\{0:0.\#\#\#\#\#\}", latitude) + " Lng:" + String.Format("\{0:0.\#\#\#\#\#\}", longitude) + "InDate:" + parse[3] + " Time:" + parse[4] + "InAcoustic:InSinyal:" + String.Format("\{0:0.\#0\}",signal/100) + " db Frek:"+ String.Format("\{0:0.\#\}",freq/100) + " Hz\nPressure:In"

String.Format("\{0:0.\#\}",pressure/100) + " Kpa Vdc:" + String.Format("\{0:0.\#\}",vdc/100) + " Vdc";

markerNode.ToolTipMode

MarkerTooltipMode.Always;

ranjauOverlay.Markers.Add(markerNode);

gMapControl1.Position = point;

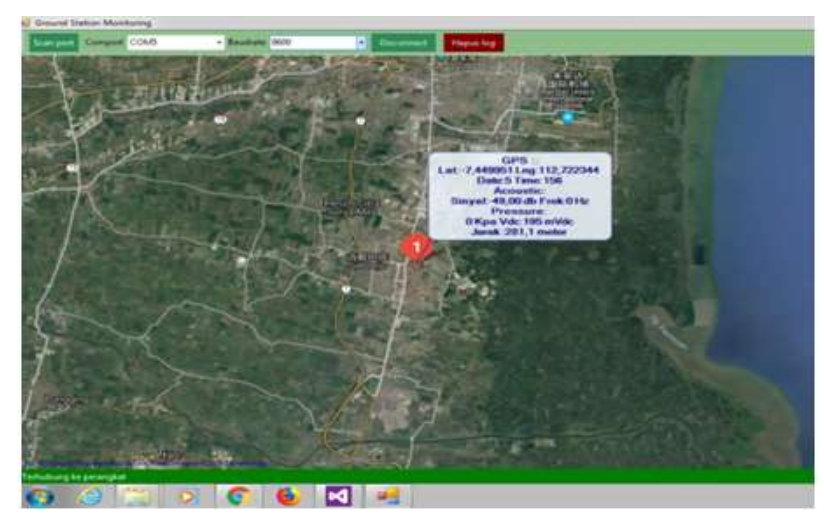

Fig.12 Electronic Map Interface View

\subsubsection{Testing Based on Distance Difference}

In Figure 13 The acceptable data acceptance proves well on Lora receivers. Testing with distance between Lora transmitter with Lora Receiver of 100 meters, data can be received on receiver side well, this is demonstrated with the emergence of ToolTip on the electronic map of ground station application, ToolTip is an interface That provide information on the location marker icon of the test mine distance 100 meters.

a. Testing at Communication Distance 100 meters

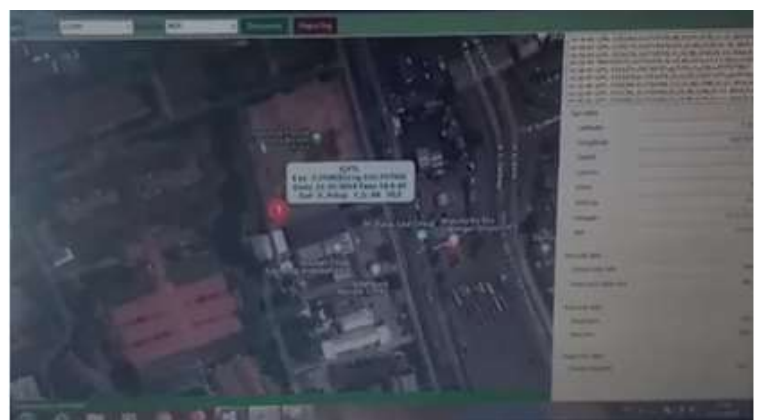

Fig.13 Communication Distance Testing Result 100 meters

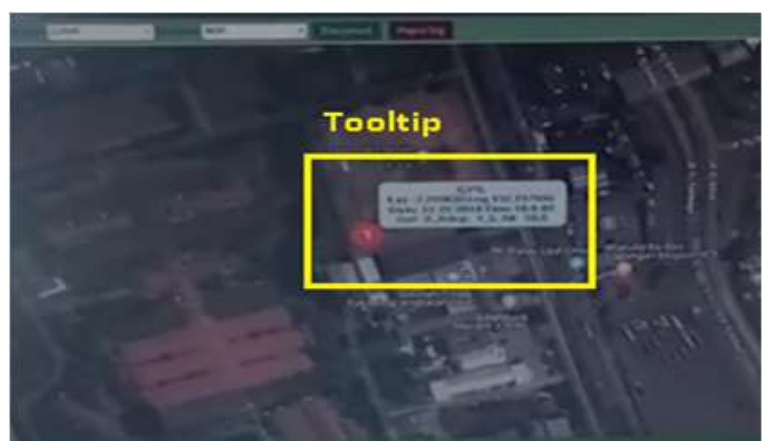

Fig.14 Interface Tooltip Success Indicator

In this test there is little obstacle. The test result can be obtained the receipt of good data communication, as shown in Figure 14 above. At the test with the distance between the Lora transmitter with a 100-metre Lora

b. Testing at Communication Distance 200 meters

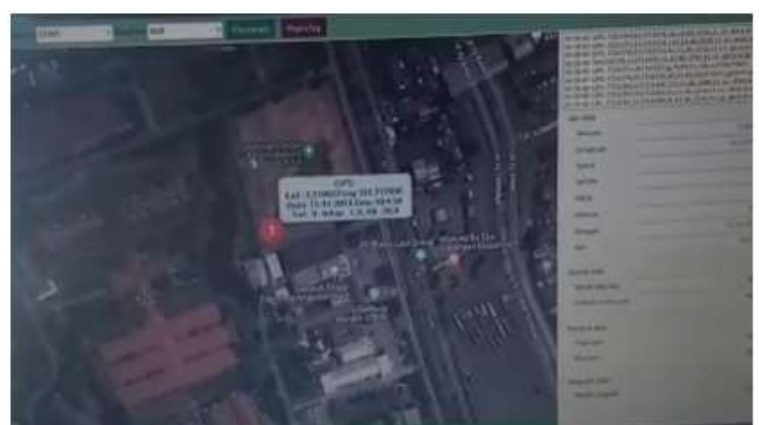

Fig.15 Communication Distance Testing Result 200 meters 


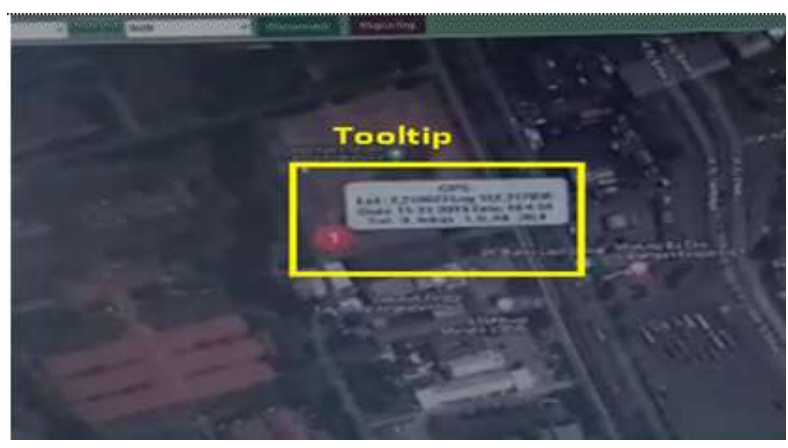

Fig.16 Interface Tooltip Success Indicator

In this test there is little obstacle. The test result can be obtained the receipt of good data communication, as shown in Figure 15 above. At the test with the distance between the Lora transmitter with a 200-metre Lora receiver, data can be received on the receiver side well, this is demonstrated with the emergence of a tooltip on the application's electronic map. In Figure 16 shows acceptable data acceptance proving well on Lora receivers.

\subsubsection{Testing With Linear Mobile Transmitter Devices}

Testing the data transmission capability with a mobile transmitter device to linear reach the author's most distant distance to get the measurement results Mobile is presented on the following image test results :

a. Mobile Data Transmission Test at a Distance of 49 meters

Obtained result of good data acceptance on ground station. Data received on ground station does not occur for packet loss. Reception Interval in range $1000 \mathrm{~ms}$. Here figure 17 Test results move at a distance of 49 meters.

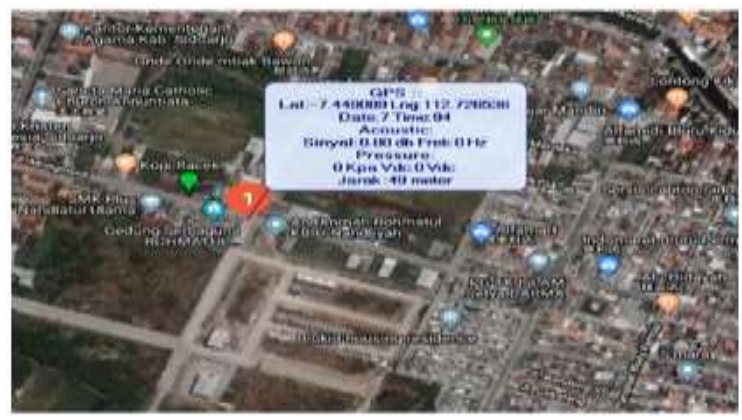

Fig.17 Mobile Test Results at a Distance of $49 \mathrm{~m}$ b. Mobile Data Transmission Test at a Distance of 112.5 meters

In Figure 18 The following shows the test results are moving at a distance of 112.5 meters. Obtained result of good data acceptance on ground station. Data received on ground station does not occur for packet loss. The receiving Interval occurred a delay of $2500 \mathrm{Ms}$.

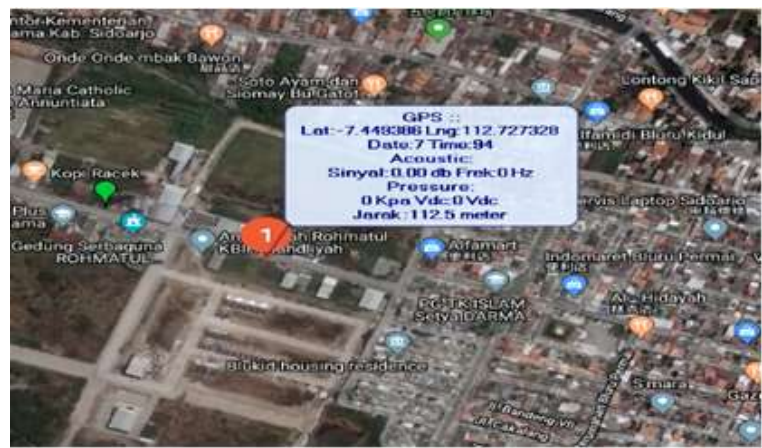

Fig.18 Mobile Test Results at a Distance of 112.5

$\mathrm{m}$

In Figure 19 The following shows the test results are moving at a distance of 195 meters. Obtained result of good data acceptance on ground station. Data received on ground station does not occur for packet loss. The receiving Interval occurred a delay of $3800 \mathrm{Ms}$.

c. Mobile Data Transmission Test at a Distance of 195 meters

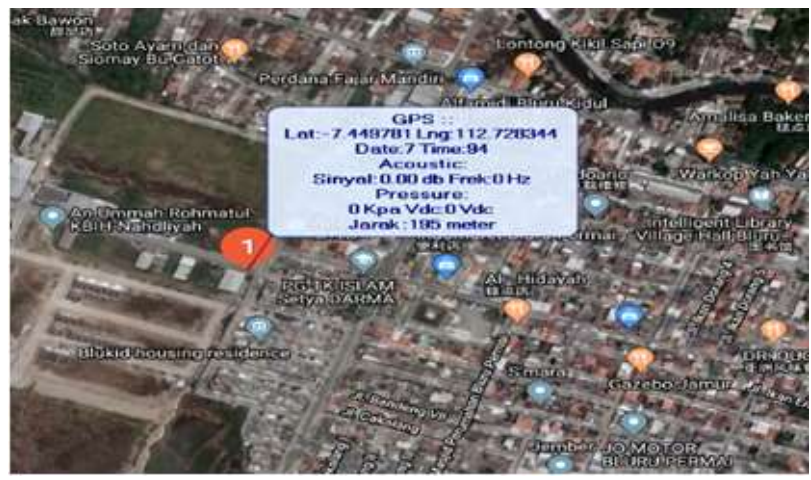

Fig.19 Mobile Test Results at a Distance of $195 \mathrm{~m}$

d. Mobile Data Transmission Test at a Distance of 222,1 meters

Obtained result of data acceptance on the ground station occurs error shown in Figure 20. The receiving Interval occurred a delay of $5000 \mathrm{Ms}$. 


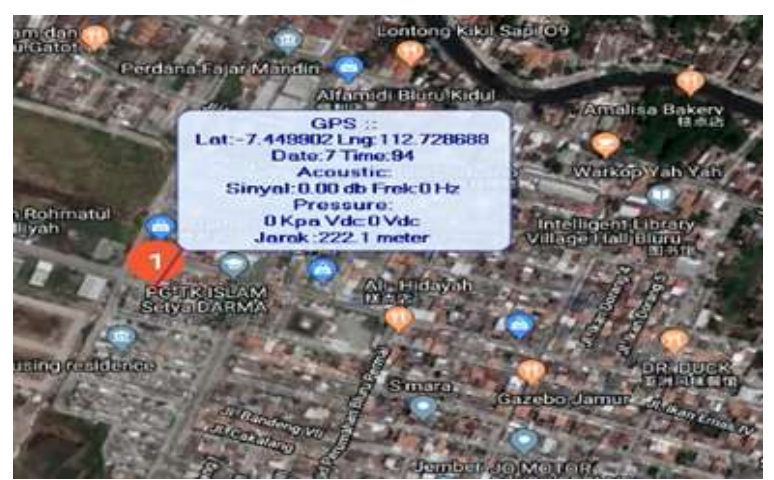

Fig.20 Mobile Test Results at a Distance of 222.1

m

\section{Acceptance Data Error}

12:9:17 GPS:-7449875,112728560,324,7,94,3200,17-11-2019,5:9:16

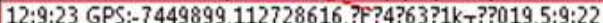
12:10:0 GPS:-7449940,112728792,50,6,107,1870,17-11-2019,5:9:59 12:10:15 GPS:-7449981,112728856,251,7,94,1940,17-11-2019,5:10:1 12:10:16 GPS:-7449982,112728864,277, $794,1910,17-11-2019,5: 10: 11$ 12:10:17 GPS:-7449986,112728872,294,7,94,1940,17-11-2019,5:10:1t 12:10:29 GPS:-7450034,112728936,262,7,107,2900,17-11-2019,5:10:: 12:10:30 GPS:-7450037,112728944,331, 7, 107,2880,17-11-2019,5:10: 12:10:43 GPS:-7450053,112729056,581,6,107,2870,17-11-2019,5:10: 12:10:46 GPS:-7450057,112729088,372,7,94,2880,17-11-2019,5:10:4! 12:11:26 GPS:-7450111,112729280,33,7,94,2170,17-11-2019,5:11:25 12:11:27 GPS:-7450112,112729280,35, 7,94,2140,17-11-2019,5:11:26 12:11:29 GPS:-7450112,112729280,117,94,2120,17-11-2019,5:11:28

Fig.21 Test Result of Receiving Data Error

\section{Data Reception Interval}

12:9:17 GPS:-7449875,112728560,324,7,94,3200,17-11-2019,5:9:16 12:9:23 GPS:-7449899, 112728616,?F?4?63?1k ???019,5:9:22 12:10:0 GPS:-7449940,112728792,50,6,107,1870,17-11-2019,5:9:59 12:10:15 GPS:-7449981,112728856,251,7,94,1940,17-11-2019,5:10:1 12:10:16 GPS:-7449982,112728864,277, 7,94,1910,17-11-2019,5:10:1! 12:10:17 GPS:-7449986,112728872,294,7,94,1940,17-11-2019,5:10:1t 12:10:29 GPS:-7450034,112728936,262,7,107,2900,17-11-2019,5:10: 12:10:30 GPS:-7450037,112728944,331,7,107,2880,17-11-2019,5:10: 12:10:43 GPS:-7450053,112729056,581,6,107,2870,17-11-2019,5:10: 12:10:46 GPS:-7450057,112729088,372, 7,94,2880,17-11-2019,5:10:4! 12:11:26 GPS:-7450111,112729280,33,7,94,2170,17-11-2019,5:11:25 12:11:27 GPS:-7450112,112729280,35,7,94,2140,17-11-2019,5:11:26 12:11:29 GPS:-7450112,112729280,11,7,94,2120,17-11-2019,5:11:28

Fig.22 Data Acceptance Delay Time Interval

As for the interval increase in reception time because of the effect of transmission distance can be explained in Figure 21. In Figure 22 described in red box is the time interval of data received on the ground station. In the first line of data received at time 12:9:17 and the next data received at 12:9:23 time, the time difference is 6 seconds, this does not correspond to the delivery time interval of 1 second set on the transmitter.
From tests gained greatly affecting distances in data transmission systems. Obstacle also greatly affects the data transmission system. Distance affects transmitter signal strength that will experience a decrease in signal strength comparable to the transmission distance in the line of side transmission mode. Obstacle can reduce signal strength on the receiver.

Obstacle with metallic base material will affect the signal strength by absorbing signal, so that the received signal will experience decreased signal strength or lost altogether. From the tests that have been made to a problem in the data transmission system is obstacle and the distance.

Adding power power to a solution to increase transmission distance. Another alternative is the use of antennas with higher gain. With moving mode or mobile omnidirectional antenna an option. In static mode the directional antenna or steering is the best choice.

On tests the author does not contribute to antenna options. Antenna used is the standard pig tail $1 \lambda$ (LAMDA) with a frequency of $433 \mathrm{MHz}$ can be obtained wavelength of $69.2 \mathrm{~cm}$. The pig tail antenna Model used is an option due to small and compact physical forms. However, pig tail antennas have weaknesses on the transmission distance side.

Power used in testing of $100 \mathrm{~mW}$ with signal strength of $18.5 \mathrm{dbm}$. Because it is the maximum power of Lora, while for sensitivity between-105 db $\mathrm{S} / \mathrm{D}-50 \mathrm{db}$. Of Lora datasheet mentioned transmission distance is able to reach $1 \mathrm{Km}$, but from the tests that have been conducted, only the maximum reach distance of $222 \mathrm{~m}$.

Found another weakness in the Lora system that is, low data rate water. For data exchange system with high data rate can not be done with Lora device. Authors have been testing with high data rate found data transmission failures. Because the maximum limit of Lora data rate is $2.4 \mathrm{Kbps}$ that 
is, 2400 bits per second of data can be transmitted. Based on the tests that have been done are comparisons with the actual measured distances shown in Table 3.1 :

Table 1. Comparison of System Distance Measurement With Actual Distance

\begin{tabular}{|c|c|c|c|c|}
\hline No & $\begin{array}{c}\text { Distance } \\
\text { System } \\
(\mathbf{m})\end{array}$ & $\begin{array}{c}\text { Actual } \\
\text { Distance } \\
(\mathbf{m})\end{array}$ & $\begin{array}{c}\text { Difference } \\
(\mathbf{m})\end{array}$ & $\begin{array}{c}\% \\
\text { Error }\end{array}$ \\
\hline 1 & 50 & 48,5 & 1,5 & 3,0 \\
\hline 2 & 75 & 78,2 & $-3,2$ & 4,3 \\
\hline 3 & 100 & 97,5 & 2,5 & 2,5 \\
\hline 4 & 125 & 130,5 & $-5,5$ & 4,4 \\
\hline 5 & 150 & 165 & -15 & 10,0 \\
\hline 6 & 175 & 182,5 & $-7,5$ & 4,3 \\
\hline 7 & 200 & 210 & -10 & 5,0 \\
\hline \multicolumn{4}{|c|}{} & \multicolumn{4}{|c|}{ Average Error \% } & 4,8 \\
\hline
\end{tabular}

Calculation of distance errors on measurements using the system compared to actual measurements, using formulas :

error $=\frac{\text { Distance system }- \text { Actual distance }}{\text { Actual distance }}$

To find the average formula used error,

average error $=\sum \frac{\text { error }}{\text { Many error }}$

Accuracy of system distance measurement obtained by accuracy :

Accuracy $=100 \%$ - average error

Accuracy $=100 \%-4,8 \%$

Accuracy $=95,2 \%$

\section{CONCLUSIONS}

From the design, testing, and discussion of research results during the preparation and manufacture of the final task can be concluded that the design of data communication systems using Lora can be obtained a maximum range of 222 meters with Condition of the building obstructed.

Based on the results of the research done then for improvement in the future, researchers suggest :

a. Develop a transmission system using an external omnidirection antenna to get a farther range.

b. Dividing the delivery data by a smaller data plan, it aims to reduce the failure rate of data transmission.

\section{REFERENCES}

Arduino 2013. About Us: Arduino. from Arduino Web site: http://arduino.cc/ en/Main/arduinoBoardMega2560.

Andrianto, Heri and Aan Darmawan (2016). Arduino Fast Learning and Programming. Bandung: Bandung Informatics.

Kadir, Abdul 2015. A Study Guide for Various Microcontroller Based Projects. Yogyakarta: ANDI Publisher.

Djuandi, F. 2011. About Us: academia.edu. Retrieved July 4, 2019, from Academia Edu Web site: https://www.academia.

NOA Labs. 2012. About Us: Smart Prototyping. Retrieved November 22, 2019, from Smart Prototyping Web site: http://www.smartprototyping.com/LoRa-SX1278-10KM-433Mlong-rangewire less-module-Ra-01. 